\title{
Exponential Attractor for Coupled Ginzburg-Landau Equations Describing Bose-Einstein Condensates and Nonlinear Optical Waveguides and Cavities
}

\author{
Gui Mu and Jun Liu \\ College of Mathematics and Information Science, Qujing Normal University, Qujing, Yunnan 655011, China \\ Correspondence should be addressed to Jun Liu; liujunxei@126.com
}

Received 4 February 2013; Accepted 6 March 2013

Academic Editor: de Dai

Copyright (C) 2013 G. Mu and J. Liu. This is an open access article distributed under the Creative Commons Attribution License, which permits unrestricted use, distribution, and reproduction in any medium, provided the original work is properly cited.

The existence of the exponential attractors for coupled Ginzburg-Landau equations describing Bose-Einstein condensates and nonlinear optical waveguides and cavities with periodic initial boundary is obtained by showing Lipschitz continuity and the squeezing property.

\section{Introduction}

Inertial set was introduced (see [1-5]) in order to overcome some of the theoretical difficulties that are associated with inertial manifolds. An inertial set, by definition, contains the global attractor and attracts all trajectories at a uniform exponential rate. Consequently, it contains the slow transients as well as the global attractor. In the theory of dynamical systems the slow transients correspond to slowly converging stable manifolds that are in some sense close to central manifolds. Numerical simulations of infinite dimensional dynamical systems often capture both slow transients and parts of the attractor. After a large but finite time the state of the system obtained from the numerical calculation may often be at a finite distance from the global attractor but at an infinitesimal distance to the inertial set. In this sense, we propose to call the inertial set an exponential attractor to be consistent with the physical intuition [5].

An exponential attractor is an exponentially attracting compact set with finite fractal dimension that is positively invariant under the forward semiflow. The notion of exponential attractors was introduced by Eden et al. [3] and has been shown to be one of the very important notions in the study of long time behavior of solutions to nonlinear diffusion equations [6]. The easiest way of obtaining an exponential attractor is by taking the intersection of an absorbing set with an inertial manifold.
In the area of hyperbolic evolutionary equations, the existence of exponential attractors has been proved for many equations. In this paper, we will prove the existence of exponential attractor for coupled Ginzburg-Landau equations

$$
\begin{gathered}
i u_{t}+\gamma_{2} \Delta u+i \gamma u+\left(\sigma_{1}+i \sigma_{2}|u|^{2}\right)|u|^{2} u+v=0 \\
i v_{t}+\gamma_{2} \Delta u+(i \Gamma-\chi) v+u=0
\end{gathered}
$$

with the periodic boundary conditions

$$
\begin{array}{r}
u(x, t)=u(x+D, t), \quad v(x, t)=v(x+D, t), \\
x \in R, \quad t>0,
\end{array}
$$

and initial value

$$
u(x, 0)=u_{0}(x), \quad v(x, 0)=v_{0}(x), \quad x \in R .
$$

Its physical realizations include systems from nonlinear optics and a double-cigar-shaped Bose-Einstein condensate with a negative scattering length. In particular, in the case of the optical systems, $u$ and $v$ are amplitudes of electromagnetic waves in two cores of the system, the evolutional variable $t$ is either time or propagation distance in the dual-core optical fiber, and $x$ is the transverse coordinate in the cavity or the reduced time in the application to the fibers [7].

This paper is organized as follows. In Section 2, we give a description of preliminaries with existence of exponential 
attractor and the properties of solutions and bounded absorbing sets of (1). In Section 3, the existence of the exponential attractor in $V_{2}$ type exponential attractor is proved. In Section 4, we give some conclusions for this paper.

\section{Preliminaries}

Let $V_{1}, V_{2}$ be two Hilbert spaces, and let $V_{2}$ be dense in $V_{1}$ and compactly imbedded into $V_{1}$. Let $S(t)_{t \geq 0}$ be a continuous map from $V_{1}, V_{2}$ into itself. We study

$$
\begin{gathered}
\frac{d u}{d t}+A u+g(u)=f(x), \quad t>0, x \in \Omega, \\
u(x, 0)=u_{0}(x),
\end{gathered}
$$

Dirichlet problem or periodic boundary problem,

where $\Omega$ is a bounded open set in $R^{n}, \partial \Omega$ is smooth, and $A$ is a positive self-adjoint operator with a compact inverse. Letting $\left\{w_{i}\right\}_{i=1}^{\infty}$ denote the complete set of eigenvectors of $A$, the corresponding eigenvalues are

$$
0 \leq \lambda_{1}<\lambda_{1} \cdots \lambda_{i}<\cdots \longrightarrow+\infty
$$

We assume that the nonlinear semigroup $S(t)_{t \geq 0}$ defined in (4)-(6) possesses a compact attractor $\mathbf{B}$ of $\left(V_{2}, V_{1}\right)$-type; namely, there exists a compact set $\mathbf{B}$ in $V_{1}$, and $\mathbf{B}$ attracts all bounded subsets in $V_{2}$ and is invariant under the action of $S(t)_{t \geq 0}$.

Let $C$ be a compact subset of $V_{2} . S(t)_{t \geq 0}$ leaves the set $C$ invariant and set

$$
\mathbf{B}=\bigcap_{s \geq 0} \overline{\bigcup_{t \geq s} S(t)_{t \geq 0} C}
$$

that is, for $S(t)_{t \geq 0}$ on $C, \mathbf{B}$ is the global attractor.

Definition 1. A compact set $M$ is called an exponential attractors for $S(t)_{t \geq 0}, C$ if

(i) $\mathbf{B} \subseteq M \subseteq C$;

(ii) $S(t) M \subseteq M$, for every $t \geq 0$;

(iii) $M$ has finite fractal dimension $d_{F}<\infty$;

(iv) There exist constants $c_{1}$ and $c_{2}$ such that

$$
\operatorname{dist}_{v_{2}}(S(t) C, M) \leq c_{1} \exp \left(-c_{2} t\right), \quad \forall t>0,
$$

where

$$
\operatorname{dist}_{v_{2}}(A, D)=\sup _{x \in A} \inf _{y \in D}\|x-y\|_{V_{2}} \text {. }
$$

Definition 2. If there exists a bounded function $l(t)$ independent $u$ and $v$ such that

$$
\|S(t) u-S(t) v\|_{V_{2}} \leq l(t)\|u-v\|_{V_{2}}
$$

for every $u, v \in C$, then we say $S(t)$ is Lipschitz continuous in $C$ and $l(t)$ is Lipschitz constant for $S(t)$ in $C$.
Definition 3. A continuous semigroup $S(t)_{t \geq 0}$ is said to satisfy the squeezing property on $C$ if there exists $t_{*}>0$ such that $S\left(t_{*}\right)$ satisfies the following.

For every $\delta \in(0,(1 / 8))$, there exists an orthogonal projection $P_{N_{0}}$ of rank equal to $N_{0}$ such that for every $u$ and $v$ in $C$ if

$$
\left\|P_{N_{0}}\left(S\left(t_{*}\right) u-S\left(t_{*}\right) v\right)\right\|_{v_{2}} \leq\left\|Q_{N_{0}}\left(S\left(t_{*}\right) u-S\left(t_{*}\right) v\right)\right\|_{v_{2}}
$$

holds, then we also have

$$
\left\|S\left(t_{*}\right) u-S\left(t_{*}\right) v\right\|_{v_{2}} \leq \delta\|u-v\|_{v_{2}}
$$

where $Q_{N_{0}}=I-P_{N_{0}}$.

Theorem 4 (see [3]). Suppose (4)-(6) satisfy the following conditions.

(1) There exist nonlinear semigroup $S(t)_{t \geq 0}$ and a compact attractor $\mathbf{B}$.

(2) There exists a compact set $\mathbf{C}$ in $V_{2}$ which is positively invariant for $S(t)_{t \geq 0}$.

(3) $S(t)_{t \geq 0}$ is Lipschitz continuous and is squeezing in $C$.

Then (4)-(6) admit an exponential attractor $M$ in $V_{2}$ for $S(t)_{t \geq 0}$ and

$$
M=\bigcup_{0 \leq t \leq t_{*}} S(t) M_{*}
$$

where

$$
M_{*}=\mathbf{B} \bigcup\left(\bigcup_{j=1}^{\infty} \bigcup_{k=1}^{\infty} S\left(t_{*}\right)^{j}\left(E^{(k)}\right)\right) .
$$

Moreover,

$$
\begin{gathered}
d_{F}(M) \leq 1+C N_{0}, \\
\operatorname{dist}_{V_{2}}(S(t) B, M) \leq C_{0} \exp \left(-C_{1} t\right),
\end{gathered}
$$

where $N_{0}, E(k)$ are defined as in [4], C, $C_{0}, C_{1}$ are the constants independent of $B$, and $t_{*}$ is a positive constant.

Proposition 5. There exists $t_{0}\left(B_{0}\right)$ such that

$$
B^{*}=\overline{\bigcup_{0 \leq t \leq t_{0}} S(t) B_{0}}
$$

is a compact positively invariant set in $V_{1}$ and is absorbing set for all bounded subsets in $V_{2}$, where $B_{0}$ is a closed absorbing set in $V_{2}$ for $S(t)_{t \geq 0}$.

Proposition 6. Let $B_{0}, B_{1}$ be bounded and closed absorbing sets for (4)-(6) in $\left(V_{2}, V_{1}\right)$, respectively. Then there exists a compact attractor $A^{*}$ of $\left(V_{2}, V_{1}\right)$-type. For the proof of Proposition 5 and Proposition 6, we refer the reader to [5].

Denoting by $|\cdot|_{L^{p}}$ the norm in $L^{p}(0, L), 1 \leq p \leq \infty$, for simplicity, we denote by $|\cdot|_{0}$ and $|\cdot|_{\infty}$ the norm in the case 
$p=2$ and $p=\infty$, respectively. Suppose that $H=L^{2}(0, L)$, $E_{i}=H^{i}(0, L) \times H^{i}(0, L)(i=1,2)$, where $H^{i}(0, L)$ is a Hilbert space for the scalar product

$$
((\cdot, \cdot))_{H^{i}}=(\cdot, \cdot)+\sum_{j=1}^{i}\left(D^{j} \cdot, D^{j} \cdot\right), \quad D=\frac{\partial}{\partial x} .
$$

The norm of $E_{i}$ is defined by $\|(u, v)\|_{E_{i}}^{2}=\|u\|_{H^{i}}^{2}+\|v\|_{H^{i}}^{2}$.

We now establish some time-uniform a priori estimates on $(u, v)$ in $E_{1}$ and $E_{2}$, respectively.

Lemma 7. Assume that $\left(u_{0}, v_{0}\right) \in E_{1}$; then

$$
\|(u, v)\|_{E_{1}}^{2} \leq c\left\|\left(u_{0}, v_{0}\right)\right\|_{E_{1}}^{2} e^{-\delta_{1} t}+c_{1} .
$$

Thus there exists $t_{1}=t_{1}(R)>0$ such that

$$
\|(u, v)\|_{E_{1}}^{2} \leq c_{2}, \quad t \geq t_{1}
$$

whenever $\left\|\left(u_{0}, v_{0}\right)\right\|_{E_{1}} \leq R$.

Lemma 8. Assume that $\left(u_{0}, v_{0}\right) \in E_{2}$; then

$$
\|(u, v)\|_{E_{2}}^{2} \leq c\left\|\left(u_{0}, v_{0}\right)\right\|_{E_{2}}^{2} e^{-\delta_{2} t}+c_{3} .
$$

Thus there exists $t_{2}=t_{2}(R)>0$ such that

$$
\|(u, v)\|_{E_{2}}^{2} \leq c_{4}, \quad t \geq t_{2},
$$

whenever $\left\|\left(u_{0}, v_{0}\right)\right\|_{E_{2}} \leq R$.

Theorem 9. Assume that all the parameters of (1) are positive. For $\left(u_{0}, v_{0}\right)$ given in $E_{i}(i=1,2)$, there exists a unique solution

$$
(u, v) \in L^{\infty}\left(R_{+}, E_{i}\right) .
$$

And also

$$
(u, v) \in \mathscr{C}\left(R_{+}, E_{1}\right), \quad \forall\left(u_{0}, v_{0}\right) \in E_{1} .
$$

Furthermore, the solution operator of the system is a continuous semigroup $S(t)$ on $E_{1}$ which possesses bounded absorbing sets $B_{i} \subset E_{i}$, for $i=1,2$.

Thus, we observe that Lemmas 7 and 8 show that there exists constant $k$ depending only on the data that the balls

$$
\begin{aligned}
& B_{1}=\left\{(u, v) \in E_{1},\|u\|_{H_{1}}+\|v\|_{H_{1}} \leq k\right\}, \\
& B_{2}=\left\{(u, v) \in E_{2},\|u\|_{H_{2}}+\|v\|_{H_{2}} \leq k\right\}
\end{aligned}
$$

are bounded absorbing sets for $S(t)$ in $E_{1}$ and $E_{2}$, respectively:

Let

$$
V_{1}=E_{1}, \quad V_{2}=E_{2}, \quad B=\overline{\bigcup_{t \geq 0} S(t) B_{2}},
$$

then $B$ is a compact invariant subset in $V_{2}$; we know that semigroup $S(t)$ defined by problem (31)-(34) possesses a $V_{2^{-}}$ type compact attractor. According to Theorem 4, we need only to show the Lipschitz continuity and the squeezing property of the dynamical system $S(t)$ in $B$, respectively. That is what we proceed to do in the following sections.

\section{Exponential Attractor in $V_{2}$ for Problem}

$$
\text { (1)-(2) }
$$

In this section, we show the existence of the exponential attractor in $V_{2}$ for problem (1)-(2). In order to prove the Lipschitz continuity and the squeezing property, we need to extend Hölder inequality

$$
\int_{\Omega}\left|u(x) u_{2}(x) \cdots u_{k}(x)\right| d_{x} \leq \prod_{j=1}^{k}\left\|u_{j}\right\|_{L^{p_{j}}},
$$

where $\sum_{j=1}^{k}\left(1 / p_{j}\right)=1, p_{j}>1$ and Gagliardo-Nirenberg (G$\mathrm{N})$ inequality

$$
\left\|\nabla^{j} u\right\|_{p} \leq c\left\|\nabla^{m} u\right\|_{r}^{a}\|u\|_{q}^{1-a}
$$

where

$$
\frac{1}{p}=\frac{j}{n}+a\left(\frac{1}{r}-\frac{m}{n}\right)+\frac{1-a}{q},
$$

$$
1 \leq q, \quad r \leq \infty, \quad 0 \leq j<m, \quad \frac{j}{m} \leq a \leq 1,
$$

and the Young's inequality

$$
\begin{gathered}
a b \leq \frac{\varepsilon}{p} a^{p}+\frac{1}{q} \varepsilon^{(-q / b)} b^{q}, \quad a, b, \varepsilon>0,1<p \\
q<\infty, \quad \frac{1}{p}+\frac{1}{q}=1 .
\end{gathered}
$$

Theorem 10. Assume $w_{1}(t)=\left(u_{1}(t), v_{1}(t)\right)$, and $w_{2}(t)=$ $\left(u_{2}(t), v_{2}(t)\right)$ are two solutions of problem (1)-(2) with initial values $w_{10}=\left(u_{10}, v_{10}\right), w_{20}=\left(u_{20}, v_{20}\right) \in B=H^{2} \times H^{2}$; then one has

$$
\left\|w_{1}(t)-w_{2}(t)\right\|_{V_{2}} \leq \exp \left(2 C_{0} t\right)\left\|w_{10}-w_{20}\right\|_{V_{2}} .
$$

Proof. Letting $h(t)=u_{1}(t)-u_{2}(t), g(t)=v_{1}(t)-v_{2}(t)$, from (1)-(2), we have

$$
\begin{gathered}
i h_{t}+\gamma_{2} \Delta h+i \gamma h+f\left(u_{1}, u_{2}\right)+g=0, \\
i g_{t}+\gamma_{2} \Delta g+(i \Gamma-\chi) g+h=0,
\end{gathered}
$$

with periodic initial value

$$
\begin{array}{r}
h(x, t)=h(x+D, t), \quad g(x, t)=g(x+D, t), \\
x \in R, \quad t>0, \\
h(x, 0)=u_{10}(x)-u_{20}(x), \quad g(x, 0)=v_{10}(x)-v_{20}(x), \\
x \in R,
\end{array}
$$

where

$$
f\left(u_{1}, u_{2}\right)=\sigma_{1}\left(\left|u_{1}\right|^{2} u_{1}-\left|u_{2}\right|^{2} u_{2}\right)+i \sigma_{2}\left(\left|u_{1}\right|^{4} u_{1}-\left|u_{2}\right|^{4} u_{2}\right) .
$$


Taking $\phi_{1}(u)=|u|^{2}$ and $\phi_{2}(u)=|u|^{4}$, then we get

$$
\begin{aligned}
& \phi_{1}^{\prime}(\xi) h=\left|u_{1}\right|^{2}-\left|u_{2}\right|^{2}, \\
& \phi_{2}^{\prime}(\eta) h=\left|u_{1}\right|^{4}-\left|u_{2}\right|^{4} .
\end{aligned}
$$

Substituting (37) and (38) into (36), we get

$$
\begin{aligned}
f\left(u_{1}, u_{2}\right)= & \sigma_{1}\left(\left|u_{1}\right|^{2} u_{1}-\left|u_{1}\right|^{2} u_{2}+\left|u_{1}\right|^{2} u_{2}-\left|u_{2}\right|^{2} u_{2}\right) \\
& +i \sigma_{2}\left(\left|u_{1}\right|^{4} u_{1}-\left|u_{1}\right|^{4} u_{1}+\left|u_{1}\right|^{4} u_{2}-\left|u_{2}\right|^{4} u_{2}\right) \\
= & \sigma_{1} h\left(\phi_{1}\left(u_{1}\right)+u_{2} \phi_{1}^{\prime}(\xi)\right) \\
& +i \sigma_{2} h\left(\phi_{2}\left(u_{1}\right)+u_{2} \phi_{2}^{\prime}(\eta)\right) .
\end{aligned}
$$

Substituting (39) into (32), we obtain

$$
\begin{gathered}
i h_{t}+\gamma_{2} \Delta h+i \gamma h+\sigma_{1} h\left(\phi_{1}\left(u_{1}\right)+u_{2} \phi_{1}^{\prime}(\xi)\right) \\
+i \sigma_{2} h\left(\phi_{2}\left(u_{1}\right)+u_{2} \phi_{2}^{\prime}(\eta)\right)+g=0, \\
\quad i g_{t}+\gamma_{2} \Delta g+(i \Gamma-\chi) g+h=0 .
\end{gathered}
$$

To prove the Theorem 4 , we take the following four steps.

Step 1 . Taking the inner product of (40) with $\bar{h}$ and (41) with $\bar{g}$, respectively, we have

$$
\begin{aligned}
\left(i h_{t}, \bar{h}\right) & +\left(\gamma_{2} \Delta h, \bar{h}\right)+(i \gamma h, \bar{h}) \\
& +\left(\sigma_{1} h\left(\phi_{1}\left(u_{1}\right)+u_{2} \phi_{1}^{\prime}(\xi)\right), \bar{h}\right) \\
& +\left(i \sigma_{2} h\left(\phi_{2}\left(u_{1}\right)+u_{2} \phi_{2}^{\prime}(\eta)\right), \bar{h}\right)+(g, \bar{h})=0, \\
\left(i g_{t}, \bar{g}\right) & +\left(\gamma_{2} \Delta g, \bar{g}\right)+((i \Gamma-\chi) g, \bar{g})+(h, \bar{g})=0,
\end{aligned}
$$

using

$$
\begin{aligned}
\frac{d}{d t} \int_{\Omega}|u|^{2} d x & =\frac{d}{d t} \int_{\Omega} u \bar{u} d x=\int_{\Omega}\left(u_{t} \bar{u}+u \overline{u_{t}}\right) d x \\
& =2 \operatorname{Re} \int_{\Omega} u_{t} \bar{u} d x .
\end{aligned}
$$

Thus,

$$
\begin{gathered}
\operatorname{Im}\left(i \int_{\Omega} u_{t} \bar{u} d x\right)=\frac{1}{2} \frac{d}{d t} \int_{\Omega}|u|^{2} d x, \\
\left(\gamma_{2} \Delta h, \bar{h}\right)=-\gamma_{2}\left\|h_{x}\right\|^{2}, \quad(i \gamma h, \bar{h})=i \gamma\|h\|^{2},
\end{gathered}
$$

then taking the imaginary part of (42) and (43), respectively,

$$
\begin{aligned}
\frac{1}{2} \frac{d}{d t}\|h\|^{2} & +\gamma\|h\|^{2}+\sigma_{1} \operatorname{Im} \int_{\Omega} u_{2} \phi_{1}^{\prime}(\xi)|h|^{2} d x \\
& +\sigma_{2} \operatorname{Im} \int_{\Omega} \phi_{2}\left(u_{1}\right)|h|^{2} d x \\
& +\sigma_{2} \operatorname{Re} \int_{\Omega} \phi_{2}^{\prime}(\eta)|h|^{2} d x+\operatorname{Im} \int_{\Omega} g \bar{h} d x=0, \\
& \frac{1}{2} \frac{d}{d t}\|g\|^{2}+\Gamma\|g\|^{2}+\operatorname{Im} \int_{\Omega} h \bar{g} d x=0,
\end{aligned}
$$

by using the extend Hölder inequality, we can obtain

$$
\left|\operatorname{Im} \int_{\Omega} g \bar{h} d x\right| \leq \frac{1}{2}\left(\|g\|^{2}+\|h\|^{2}\right),
$$

$\left.\left.\left|\sigma_{1} \operatorname{Im} \int_{\Omega} u_{2} \phi_{1}^{\prime}(\xi)\right| h\right|^{2} d x|\leq| \sigma_{1}\left|\int_{\Omega}\right| u_{2}\left|\phi_{1}^{\prime}(\xi)\right| h\right|^{2} d x$

$$
\begin{aligned}
& \leq\left|\sigma_{1}\right|\|h\|^{2}\left\|u_{2}\right\|_{\infty}\left\|\phi_{1}^{\prime}(\xi)\right\|_{\infty} \\
& \leq C\|h\|^{2},
\end{aligned}
$$

$\left.\left.\left|\sigma_{2} \operatorname{Re} \int_{\Omega} u_{2} \phi_{2}^{\prime}(\eta)\right| h\right|^{2} d x|\leq| \sigma_{2}\left|\int_{\Omega}\right| u_{2}\left|\phi_{2}^{\prime}(\xi)\right| h\right|^{2} d x$ $\leq\left|\sigma_{2}\right|\|h\|^{2}\left\|u_{2}\right\|_{\infty}\left\|\phi_{2}^{\prime}(\eta)\right\|_{\infty}$ $\leq C\|h\|^{2}$.

Combining (46) and (47), then we infer that

$$
\begin{aligned}
& \frac{1}{2} \frac{d}{d t}\left(\|h\|^{2}+\|g\|^{2}\right)+\gamma\|h\|^{2}+\gamma\|g\|^{2} \\
& \quad+\sigma_{2} \int_{\Omega} \phi_{2}\left(u_{1}\right)|h|^{2} d x \leq C\|h\|^{2}+\|g\|^{2} .
\end{aligned}
$$

Step 2. Taking the inner product of (40) with $-\overline{h_{x x}}$ and (41) with $-\overline{g_{x x}}$, respectively, we have

$$
\begin{aligned}
\left(i h_{t}, \overline{h_{x x}}\right) & +\left(\gamma_{2} \Delta h,-\overline{h_{x x}}\right)+\left(i \gamma h,-\overline{h_{x x}}\right) \\
& +\left(\sigma_{1} h\left(\phi_{1}\left(u_{1}\right)+u_{2} \phi_{1}^{\prime}(\xi)\right),-\overline{h_{x x}}\right) \\
& +\left(i \sigma_{2} h\left(\phi_{2}\left(u_{1}\right)+u_{2} \phi_{2}^{\prime}(\eta)\right),-\overline{h_{x x}}\right) \\
& +\left(g,-\overline{h_{x x}}\right)=0,
\end{aligned}
$$

$$
\begin{aligned}
\left(i g_{t},-\overline{g_{x x}}\right) & +\left(\gamma_{2} \Delta g,-\overline{g_{x x}}\right)+\left((i \Gamma-\chi) g,-\overline{g_{x x}}\right) \\
& +\left(h,-\overline{g_{x x}}\right)=0 .
\end{aligned}
$$

Note that

$$
\begin{gathered}
\left(i h_{t}, \overline{h_{x x}}\right)=i \int_{\Omega} h_{x t} \overline{h_{x}} d x, \\
\left(g,-\overline{h_{x x}}\right)=\int_{\Omega} g_{x} \overline{h_{x}} d x, \\
\left(\gamma_{2} \Delta h, \overline{h_{x x}}\right)=\|\Delta h\|^{2}, \\
\left(i \gamma h,-\overline{h_{x x}}\right)=i \gamma\left\|h_{x}\right\|^{2},
\end{gathered}
$$




$$
\begin{gathered}
\left(\sigma_{1} h\left(\phi_{1}\left(u_{1}\right)+u_{2} \phi_{1}^{\prime}(\xi)\right), \overline{h_{x x}}\right) \\
=\sigma_{1} \int_{\Omega}\left[\left|h_{x}\right|^{2}\left(\phi_{1}\left(u_{1}\right)+u_{2} \phi_{1}^{\prime}(\xi)\right)\right. \\
\left.+h \overline{h_{x}}\left(\phi_{1}\left(u_{1}\right)+u_{2} \phi_{1}^{\prime}(\xi)\right)_{x}\right] d x, \\
\left(i \sigma_{2} h\left(\phi_{2}\left(u_{1}\right)+u_{2} \phi_{2}^{\prime}(\eta)\right),-\overline{h_{x x}}\right) \\
=i \sigma_{2} \int_{\Omega}\left[\left|h_{x}\right|^{2}\left(\phi_{2}\left(u_{1}\right)+u_{2} \phi_{2}^{\prime}(\eta)\right)\right. \\
\left.\quad+h \overline{h_{x}}\left(\phi_{2}\left(u_{1}\right)+u_{2} \phi_{2}^{\prime}(\eta)\right)_{x}\right] d x,
\end{gathered}
$$

then taking the imaginary part of (50) and (51), respectively,

$$
\begin{aligned}
& \frac{1 \frac{d}{d t}\left\|h_{x}\right\|^{2}+\gamma\left\|h_{x}\right\|^{2}}{+} \\
& +\sigma_{1} \operatorname{Im} \int_{\Omega}\left(u_{2} \phi_{1}^{\prime}(\xi)\left|h_{x}\right|^{2}\right. \\
& \left.\quad+h \overline{h_{x}}\left(\phi_{1}\left(u_{1}\right)+u_{2} \phi_{1}^{\prime}(\xi)\right)_{\mathrm{x}}\right) d x \\
& +\sigma_{2} \operatorname{Im} \int_{\Omega} \phi_{2}\left(u_{1}\right)\left|h_{x}\right|^{2} d x \\
& +\sigma_{2} \operatorname{Re} \int_{\Omega}\left(u_{2} \phi_{2}^{\prime}(\eta)\left|h_{x}\right|^{2}\right. \\
& \left.\quad+h \overline{h_{x}}\left(\phi_{2}\left(u_{1}\right)+u_{2} \phi_{2}^{\prime}(\eta)\right)_{x}\right) d x \\
& +\operatorname{Im} \int_{\Omega} g_{x} \overline{h_{x}} d x=0, \\
& \quad \frac{1}{2} \frac{d}{d t}\|g\|^{2}+\Gamma\|g\|^{2}+\operatorname{Im} \int_{\Omega} h \bar{g} d x=0 .
\end{aligned}
$$

Note the following inequalities:

$$
\begin{gathered}
\left|\sigma_{1} \operatorname{Im} \int_{\Omega}\left(u_{2} \phi_{1}^{\prime}(\xi)\left|h_{x}\right|^{2}+h \overline{h_{x}}\left(\phi_{1}\left(u_{1}\right)+u_{2} \phi_{1}^{\prime}(\xi)\right)_{x}\right) d x\right| \\
\leq\left|\sigma_{1}\right| \operatorname{Im} \int_{\Omega}\left(\left|u_{2}\right|\left|\phi_{1}^{\prime}(\xi)\right|\left|h_{x}\right|^{2}\right. \\
+|h|\left|\overline{h_{x}}\right|\left(\left|\phi_{1}\left(u_{1}\right)_{x}\right|+\left|u_{2 x}\right|\left|\phi_{1}^{\prime}(\xi)\right|\right. \\
\left.\left.+\left|u_{2}\right|\left|\phi_{1}^{\prime}(\xi)_{x}\right|\right)\right) d x
\end{gathered}
$$

$\leq C\left\|h_{x}\right\|^{2}+\left|\sigma_{1}\right|\|h\|\left\|\overline{h_{x}}\right\|\left(\left\|\phi_{1}\left(u_{1}\right)_{x}\right\|_{\infty}+\left\|u_{2 x}\right\|_{\infty}\left\|\phi_{1}^{\prime}(\xi)\right\|_{\infty}\right.$

$$
\left.+\left\|u_{2}\right\|_{\infty}\left\|\phi_{1}^{\prime}(\xi)_{x}\right\|_{\infty}\right)
$$

$\leq C\left\|h_{x}\right\|^{2}+c\|h\|^{2}$,

$\sigma_{2} \operatorname{Re} \int_{\Omega}\left(u_{2} \phi_{2}^{\prime}(\eta)\left|h_{x}\right|^{2}+h \overline{h_{x}}\left(\phi_{2}\left(u_{1}\right)+u_{2} \phi_{2}^{\prime}(\eta)\right)_{x}\right) d x$

$\leq C\left\|h_{x}\right\|^{2}+c\|h\|^{2}$,
Combining (53) and (54), one can obtain

$$
\begin{aligned}
& \frac{1}{2} \frac{d}{d t}\left(\left\|h_{x}\right\|^{2}+\left\|g_{x}\right\|^{2}\right)+\gamma\left\|h_{x}\right\|^{2}+\gamma\left\|g_{x}\right\|^{2} \\
& \quad+\sigma_{2} \int_{\Omega} \phi_{2}\left(u_{1}\right)\left|h_{x}\right|^{2} d x \leq C\left\|h_{x}\right\|^{2}+\left\|g_{x}\right\|^{2}+c\|h\|^{2} .
\end{aligned}
$$

Step 3. Taking the inner product of (40) with $\overline{h_{x x x x}}$ and (41) with $\overline{g_{x x x x}}$, respectively, we have

$$
\begin{aligned}
\left(i h_{t}, \overline{h_{x x x x}}\right) & +\left(\gamma_{2} \Delta h, \overline{h_{x x x x}}\right)+\left(i \gamma h, \overline{h_{x x x x}}\right) \\
& +\left(\sigma_{1} h\left(\phi_{1}\left(u_{1}\right)+u_{2} \phi_{1}^{\prime}(\xi)\right), \overline{h_{x x x x}}\right) \\
& +\left(i \sigma_{2} h\left(\phi_{2}\left(u_{1}\right)+u_{2} \phi_{2}^{\prime}(\eta)\right), \overline{h_{x x x x}}\right) \\
& +\left(g, \overline{h_{x x x x}}\right)=0, \\
\left(i g_{t}, \overline{g_{x x x x}}\right) & +\left(\gamma_{2} \Delta g, \overline{g_{x x x x}}\right)+\left((i \Gamma-\chi) g, \overline{g_{x x x x}}\right) \\
& +\left(h, \overline{g_{x x x x}}\right)=0,
\end{aligned}
$$

using

$$
\begin{gathered}
\left(i h_{t}, \overline{h_{x x x x}}\right)=i \int_{\Omega} h_{x x t} \overline{h_{x x}} d x, \\
\left(g, \overline{h_{x x x x}}\right)=\int_{\Omega} g_{x x} \overline{h_{x x}} d x, \\
\left(\gamma_{2} \Delta h, \overline{h_{x x x x}}\right)=\left\|h_{x x x}\right\|^{2}, \\
\left(i \gamma h, \overline{h_{x x x x}}\right)=i \gamma\left\|h_{x x}\right\|^{2}, \\
\left(\sigma_{1} h\left(\phi_{1}\left(u_{1}\right)+u_{2} \phi_{1}^{\prime}(\xi)\right), \overline{h_{x x x x}}\right) \\
=\sigma_{1}\left(\left(h\left(\phi_{1}\left(u_{1}\right)+u_{2} \phi_{1}^{\prime}(\xi)\right)\right)_{x x}, \overline{h_{x x}}\right) \\
=\sigma_{1}\left(h_{x x} \phi_{1}\left(u_{1}\right)+\psi_{1}, \overline{h_{x x}}\right), \\
\left(i \sigma_{2} h\left(\phi_{2}\left(u_{1}\right)+u_{2} \phi_{2}^{\prime}(\eta)\right), \overline{h_{x x x x}}\right) \\
=i \sigma_{2}\left(\left(h\left(\phi_{2}\left(u_{1}\right)+u_{2} \phi_{2}^{\prime}(\eta)\right)\right)_{x x}, \overline{h_{x x}}\right) \\
=i \sigma_{2}\left(h_{x x} \phi_{2}\left(u_{1}\right)+\psi_{2}, \overline{h_{x x}}\right),
\end{gathered}
$$

where

$$
\begin{aligned}
\psi_{1}= & h_{x x} u_{2} \phi_{1}^{\prime}(\xi) \\
& +2 h_{x}\left(\phi_{1}\left(u_{1}\right)_{x}+u_{2 x} \phi_{1}^{\prime}(\xi)+u_{2} \phi_{1}^{\prime}(\xi)_{x}\right) \\
& +h\left(\phi_{1}\left(u_{1}\right)_{x x}+u_{2 x x} \phi_{1}^{\prime}(\xi)+2 u_{2 x} \phi_{1}^{\prime}(\xi)_{x}\right. \\
& \left.+u_{2} \phi_{1}^{\prime}(\xi)_{x x}\right),
\end{aligned}
$$




$$
\begin{aligned}
\psi_{2}= & h_{x x} u_{2} \phi_{2}^{\prime}(\eta) \\
& +2 h_{x}\left(\phi_{2}\left(u_{1}\right)_{x}+u_{2 x} \phi_{2}^{\prime}(\eta)+u_{2} \phi_{2}^{\prime}(\eta)_{x}\right) \\
& +h\left(\phi_{2}\left(u_{1}\right)_{x x}+u_{2 x x} \phi_{2}^{\prime}(\eta)+2 u_{2 x} \phi_{2}^{\prime}(\eta)_{x}\right. \\
& \left.+u_{2} \phi_{2}^{\prime}(\eta)_{x x}\right),
\end{aligned}
$$

then taking the imaginary part of (50) and (51), respectively,

$$
\begin{aligned}
& \frac{1}{2} \frac{d}{d t}\left\|h_{x x}\right\|^{2}+\gamma\left\|h_{x x}\right\|^{2}+\sigma_{1} \operatorname{Im}\left(\psi_{1}, \overline{h_{x x}}\right) \\
&+\sigma_{2}\left(h_{x x} \phi_{2}\left(u_{1}\right), \overline{h_{x x}}\right)+\sigma_{2} \operatorname{Re}\left(\psi_{2}, \overline{h_{x x}}\right) \\
&+\operatorname{Im} \int_{\Omega} g_{x x} \overline{h_{x x}} d x=0 \\
& \frac{1}{2} \frac{d}{d t}\left\|g_{x x}\right\|^{2}+\Gamma\left\|g_{x x}\right\|^{2}+\operatorname{Im} \int_{\Omega} h_{x x} \overline{g_{x x}} d x=0 .
\end{aligned}
$$

Note the following inequalities:

$$
\left|\operatorname{Im}\left(\psi_{1}, \overline{h_{x x}}\right)\right| \leq C\|h\|_{H^{2}}^{2}, \quad\left|\sigma_{2} \operatorname{Re}\left(\psi_{2}, \overline{h_{x x}}\right)\right| \leq C\|h\|_{H^{2}}^{2} .
$$

Combining (60) and (61), one can obtain

$$
\begin{aligned}
& \frac{1}{2} \frac{d}{d t}\left(\left\|h_{x x}\right\|^{2}+\left\|g_{x x}\right\|^{2}\right)+\gamma\left\|h_{x x}\right\|^{2}+\Gamma\left\|g_{x x}\right\|^{2} \\
& \quad+\sigma_{2} \int_{\Omega} \phi_{2}\left(u_{1}\right)\left|h_{x x}\right|^{2} d x \leq C\|h\|_{H^{2}}^{2}+\left\|g_{x x}\right\|^{2} .
\end{aligned}
$$

Step 4. Combining (49), (56) and (63), we get

$$
\begin{aligned}
\frac{1}{2} \frac{d}{d t}( & \left.\|h\|_{H^{2}}^{2}+\|g\|_{H^{2}}^{2}\right)+\gamma\|h\|_{H^{2}}^{2}+\gamma\|g\|_{H^{2}}^{2} \\
& +\sigma_{2} \int_{\Omega} \phi_{2}\left(u_{1}\right)\left(|h|^{2}+\left|h_{x}\right|^{2}+\left|h_{x x}\right|^{2}\right) d x \\
\leq & C\left(\|h\|^{2}+\left\|h_{x}\right\|^{2}+\|h\|_{H^{2}}^{2}\right)+\|g\|^{2}+\left\|g_{x}\right\|^{2}+\left\|g_{x x}\right\|^{2} .
\end{aligned}
$$

Taking $\mu=\min (\Gamma, \gamma), C_{0}=\max (C, 1)$ and noting that

$$
\sigma_{2} \int_{\Omega}\left(|h|^{2}+\left|h_{x}\right|^{2}+\left|h_{x x}\right|^{2}\right) d x \geq 0
$$

so (64) can be reduced to

$$
\begin{aligned}
& \frac{1}{2} \frac{d}{d t}\left(\|h\|_{H^{2}}^{2}+\|g\|_{H^{2}}^{2}\right)+\mu\left(\|h\|_{H^{2}}^{2}+\|g\|_{H^{2}}^{2}\right) \\
& \leq C_{0}\left(\|h\|_{H^{2}}^{2}+\|g\|_{H^{2}}^{2}\right) .
\end{aligned}
$$

By Gronwall's inequality

$$
\|h\|_{H^{2}}^{2}+\|g\|_{H^{2}}^{2} \leq \exp \left(2 C_{0} t\right)\left(\|h(0)\|_{H^{2}}^{2}+\|g(0)\|_{H^{2}}^{2}\right),
$$

that is,

$$
\left\|w_{1}(t)-w_{2}(t)\right\|_{V_{2}} \leq \exp \left(2 C_{0} t\right)\left\|w_{10}-w_{20}\right\|_{V_{2}} .
$$

Meanwhile, it indicates that the Lipschitz constant $l(t) \leq$ $\exp \left(2 C_{0} t\right)$. This completes the proof.

Now, we intend to show the squeezing property for semigroup $S(t)$. To this end, we introduce the operator $A=$ $-\left(\partial / \partial x^{2}\right)$ from $D(A)$ to $H$ with domain

$$
D(A)=\left\{u \in H^{2}(\Omega)\right\} .
$$

Obviously, $A$ is an unbounded self-adjoint positive operator and the inverse $A^{-1}$ is compact. Thus, there exists an orthonormal basis $\left\{w_{i}\right\}_{i=1}^{\infty} i=1$ of $H$ consisting of eigenvectors of $A$ such that

$$
\begin{gathered}
A w_{i}=\lambda_{i} w_{i}, \\
0 \leq \lambda_{1}<\lambda_{1} \cdots \lambda_{i}<\cdots \longrightarrow+\infty, \quad \text { when } i \longrightarrow \infty .
\end{gathered}
$$

For all $N$ denote by $P=P_{n}: H \rightarrow \operatorname{span}\left\{w_{1}, w_{2}, \ldots, w_{n}\right\}$ the projector $Q=Q_{N}=I-P_{N}$. In the following, we will use

$$
\begin{gathered}
\left\|A^{(1 / 2)} u\right\|=\left\|\frac{\partial u}{\partial x}\right\|, \\
\left\|A^{(1 / 2)} u\right\| \geq \lambda_{N+1}^{(1 / 2)}, \quad u \in Q_{N} H, \\
\left\|Q_{N} u\right\| \leq\|u\|, \quad u \in H, \\
\left\|A Q_{N} u\right\|=\left\|Q_{N} A u\right\| \leq\|A u\|, \quad u \in D(A) .
\end{gathered}
$$

Decompose $h, g$ as

$$
h=P h+Q h, \quad g=P g+Q g .
$$

Applying $Q$ to (32) and (33) we find that

$$
\begin{gathered}
i Q h_{t}+\gamma_{2} \Delta Q h+i \gamma h+Q f\left(u_{1}, u_{2}\right)+Q g=0, \\
i Q g_{t}+\gamma_{2} \Delta Q g+(i \Gamma-\chi) Q g+Q h=0 .
\end{gathered}
$$

Take the inner product of (73) with $\overline{Q h}$ and (74) with $\overline{Q g}$, respectively. Then like Step 1, we can get

$$
\begin{gathered}
\frac{1}{2} \frac{d}{d t}\left(\|Q h\|^{2}+\|Q g\|^{2}\right)+\gamma\|Q h\|^{2}+\Gamma\|Q g\|^{2} \\
+\sigma_{2} \int_{\Omega} Q \phi_{2}\left(u_{1}\right)|Q h|^{2} d x \\
\leq C\|Q h\|^{2}+\|Q g\|^{2} .
\end{gathered}
$$

Take the inner product of (73) with $-\overline{\mathrm{Qh}}$ an and (74) with $-\overline{Q g_{x x}}$, respectively. Then like Step 2, we can get

$$
\begin{gathered}
\frac{1}{2} \frac{d}{d t}\left(\left\|Q h_{x}\right\|^{2}+\left\|Q g_{x}\right\|^{2}\right)+\gamma\left\|Q h_{x}\right\|^{2}+\Gamma\left\|Q g_{x}\right\|^{2} \\
+\sigma_{2} \int_{\Omega} Q \phi_{2}\left(u_{1}\right)\left|Q h_{x}\right|^{2} d x \\
\leq C\left\|Q h_{x}\right\|^{2}+\left\|Q g_{x}\right\|^{2}+c\|Q h\|^{2} .
\end{gathered}
$$


Take the inner product of (73) with $\overline{Q h_{x x x x}}$ and (74) with $\overline{\mathrm{Q} g_{x x x x}}$, respectively. Then like Step 3, we can get

$$
\begin{gathered}
\frac{1}{2} \frac{d}{d t}\left(\left\|Q h_{x x}\right\|^{2}+\left\|Q g_{x x}\right\|^{2}\right)+\gamma\left\|Q h_{x x}\right\|^{2}+\Gamma\left\|Q g_{x x}\right\|^{2} \\
+\sigma_{2} \int_{\Omega} Q \phi_{2}\left(u_{1}\right)\left|Q h_{x x}\right|^{2} d x \\
\leq C\|Q h\|_{H^{2}}^{2}+\left\|Q g_{x x}\right\|^{2} .
\end{gathered}
$$

Combining (75), (76), and (77), we get

$$
\begin{aligned}
& \frac{1}{2} \frac{d}{d t}\left(\|Q h\|_{H^{2}}^{2}+\|Q g\|_{H^{2}}^{2}\right)+\mu\left(\|Q h\|_{H^{2}}^{2}+\|Q g\|_{H^{2}}^{2}\right) \\
& \leq C_{0}\left(\|Q h\|_{H^{2}}^{2}+\|Q g\|_{H^{2}}^{2}\right) .
\end{aligned}
$$

Using the G-N inequality

$$
\left\|u_{x}\right\|^{2} \leq\|u\|\left\|u_{x x}\right\| \leq \frac{1}{2}\left(\|u\|^{2}+\left\|u_{x x}\right\|^{2}\right),
$$

from (78), we have

$$
\begin{aligned}
\frac{1}{2} \frac{d}{d t} & \left(\|Q h\|_{H^{2}}^{2}+\|Q g\|_{H^{2}}^{2}\right)+\mu\left(\|Q h\|_{H^{2}}^{2}+\|Q g\|_{H^{2}}^{2}\right) \\
& \leq \frac{3 C_{0}}{2}\left(\|Q h\|+\left\|Q h_{x x}\right\|+\|Q g\|+\left\|Q g_{x x}\right\|\right) \\
& \leq \frac{3 C_{0}}{2} \lambda_{N+1}^{-1}\left(\left\|Q h_{x x}\right\|+\left\|Q g_{x x}\right\|\right) \\
& \leq \frac{3 C_{0}}{2} \lambda_{N+1}^{-1}\left(\|Q h\|_{H^{2}}^{2}+\|Q g\|_{H^{2}}^{2}\right) \\
& \leq \frac{3 C_{0}}{2} \lambda_{N+1}^{-1} \exp \left(2 C_{0} t\right)\left(\|h(0)\|_{H^{2}}^{2}+\|g(0)\|_{H^{2}}^{2}\right)
\end{aligned}
$$

By Gronwall lemma we get

$$
\begin{aligned}
\|Q h\|_{H^{2}}^{2}+\|Q g\|_{H^{2}}^{2} & \\
\leq & \left(\|h(0)\|_{H^{2}}^{2}+\|g(0)\|_{H^{2}}^{2}\right) \exp (-2 \mu t) \\
& +\bar{C} \lambda_{N+1}^{-1} \exp \left(2 C_{0} t\right)\left(\|h(0)\|_{H^{2}}^{2}+\|g(0)\|_{H^{2}}^{2}\right) \\
\leq & {\left[\exp (-2 \mu t)+\bar{C} \lambda_{N+1}^{-1} \exp \left(2 C_{0} t\right)\right] } \\
& \times\left(\|h(0)\|_{H^{2}}^{2}+\|g(0)\|_{H^{2}}^{2}\right) .
\end{aligned}
$$

Letting $t_{*}>0$ be fixed we take $w(t)=w_{1}(t)-w_{2}(t)=$ $(h(t), g(t))$ and assume that

$$
\exp \left(-2 \mu t_{*}\right) \leq \frac{1}{256} .
$$

Then we choose $N$ large enough so that

$$
\bar{C} \lambda_{N+1}^{-1} \exp \left(2 C_{0} t\right) \leq \frac{1}{256},
$$

that is,

$$
\lambda_{N+1} \geq 256 \bar{C} \exp \left(2 C_{0} t\right)
$$

From (82) and (84), we obtain

$$
\|Q h\|_{H^{2}}^{2}+\|Q g\|_{H^{2}}^{2} \leq \frac{1}{128}\left(\|h(0)\|_{H^{2}}^{2}+\|g(0)\|_{H^{2}}^{2}\right) .
$$

This shows that when $t_{*}>0$ is fixed, Lipschitz constant for $S(t)$ in $B$ is equal to $\exp \left(2 C_{0} t_{*}\right)$ and $N$ satisfies

$$
\lambda_{N+1} \geq 256 \bar{C} \exp \left(2 C_{0} t_{*}\right) \text {. }
$$

We have

$$
\|Q w\|_{V_{2}} \leq\|Q w(0)\|_{V_{2}}
$$

So when

$$
\begin{aligned}
& \left\|Q w\left(t_{*}\right)\right\|_{V_{2}}>\left\|P w\left(t_{*}\right)\right\|_{V_{2}}, \\
& \left\|w\left(t_{*}\right)\right\|_{V_{2}}=\left\|Q w\left(t_{*}\right)\right\|_{V_{2}}+\left\|P w\left(t_{*}\right)\right\|_{V_{2}} \\
& <2\left\|Q w\left(t_{*}\right)\right\|_{V_{2}} \leq \frac{1}{64}\|Q w(0)\|_{V_{2}} \\
& \leq \frac{1}{64}\|w(0)\|_{V_{2}} .
\end{aligned}
$$

This completes the proof of Theorem 4 .

Theorem 11. The semigroup $S(t)$ associated with problem (1)(2) is squeezing in B. Now we conclude this section by giving our main result.

Theorem 12. Suppose that problem (1)-(2) satisfies Theorem 9; there exist $t_{*} \geq(1 / 2 \mu) \ln (256)$ and $N$ large enough such that

$$
\lambda_{N+1} \geq 256 \bar{C} \exp \left(2 C_{0} t_{*}\right) .
$$

Then for the nonlinear semigroup $S(t)$ defined in (4) and (5), $S(t)_{t \leq 0} ; B$ admits an exponential attractor $M$ in $V_{2}$ and

$$
\begin{gathered}
d_{F}(M) \leq 1+C N_{0}, \\
\operatorname{dist}_{V_{2}}(S(t) B, M) \leq C_{0} \exp \left(-C_{1} t\right),
\end{gathered}
$$

where $C_{0}, C_{1}, C$ are constants independent of the solution of the equation.

\section{Conclusions}

In this paper, we have studied the coupled Ginzburg-Landau equations which describe Bose-Einstein condensates and nonlinear optical waveguides and cavities with periodic initial boundary; the existence of the exponential attractors is obtained by showing Lipschitz continuity and the squeezing property. For exponential attractor, $N$ is only large enough such that

$$
\lambda_{N+1} \geq 256 \bar{C} \exp \left(2 C_{0} t_{*}\right) .
$$

\section{Acknowledgment}

This work was supported by Chinese Natural Science Foundation Grant no. 11061028. 


\section{References}

[1] I. S. Aranson and L. Kramer, "The world of the complex Ginzburg-Landau equation.," Reviews of Modern Physics, vol. 74, no. 1, pp. 99-143, 2002.

[2] B. A. Malomed, "Complex Ginzburg-Landau equation," in Encyclopedia of Nonlinear Science, A. Scott, Ed., pp. 157-160, Routledge, New York, NY, USA, 2005.

[3] A. Eden, C. Foias, B. Nicolaenko, and K. Temam, Exponential Attractors for Dissipative Evolution Equations, vol. 37, John Wiley, New York, NY, USA, 1994.

[4] Z. Dai and B. Guo, "Inertial fractal sets for dissipative Zakharov system," Acta Mathematicae Applicatae Sinica, vol. 13, no. 3, pp. 279-288, 1997.

[5] Z. Dai and B. Guo, Inertial ManiFolds and Approximate Inertial Mani-Folds, Science Press, Beijing, China, 2000.

[6] Z. Dai, Y. Huang, and X. Sun, "Long-time behavior of solution for coupled Ginzburg-Landau equations describing BoseEinstein condensates and nonlinear optical waveguides and cavities," Journal of Mathematical Analysis and Applications, vol. 362, no. 1, pp. 125-139, 2010.

[7] H. Sakaguchi and B. A. Malomed, "Stable solitons in coupled Ginzburg-Landau equations describing Bose-Einstein condensates and nonlinear optical waveguides and cavities," Physica D, vol. 183 , no. 3-4, pp. 282-292, 2003. 


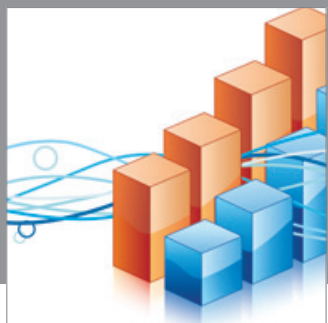

Advances in

Operations Research

mansans

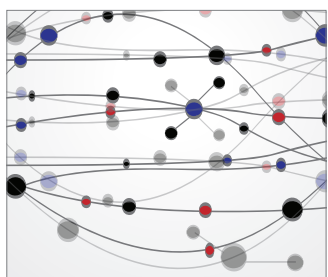

The Scientific World Journal
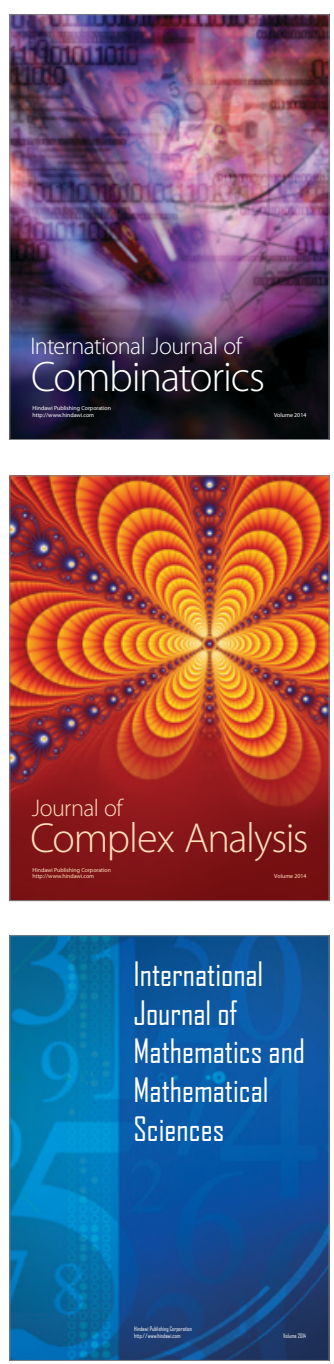
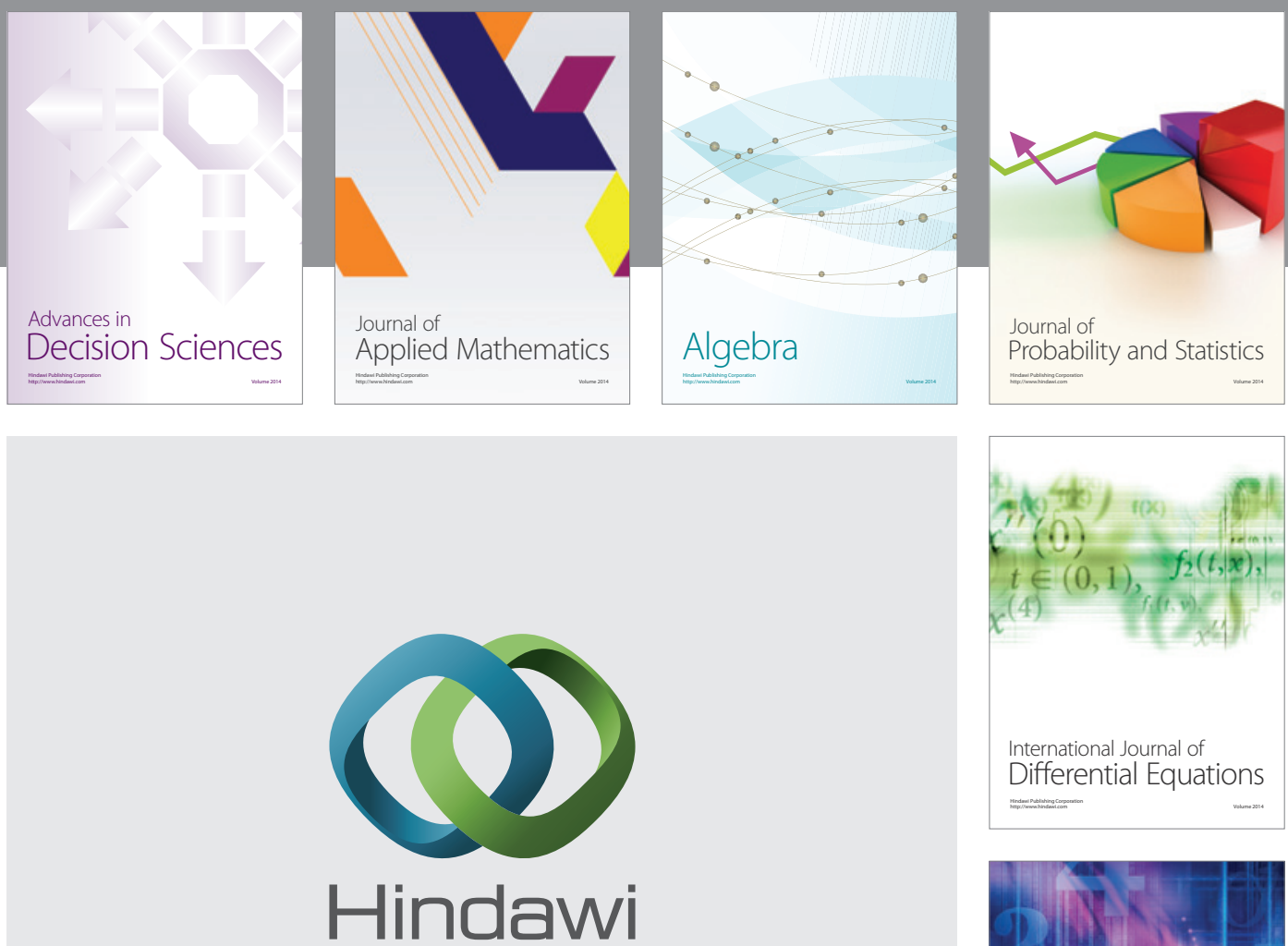

Submit your manuscripts at http://www.hindawi.com
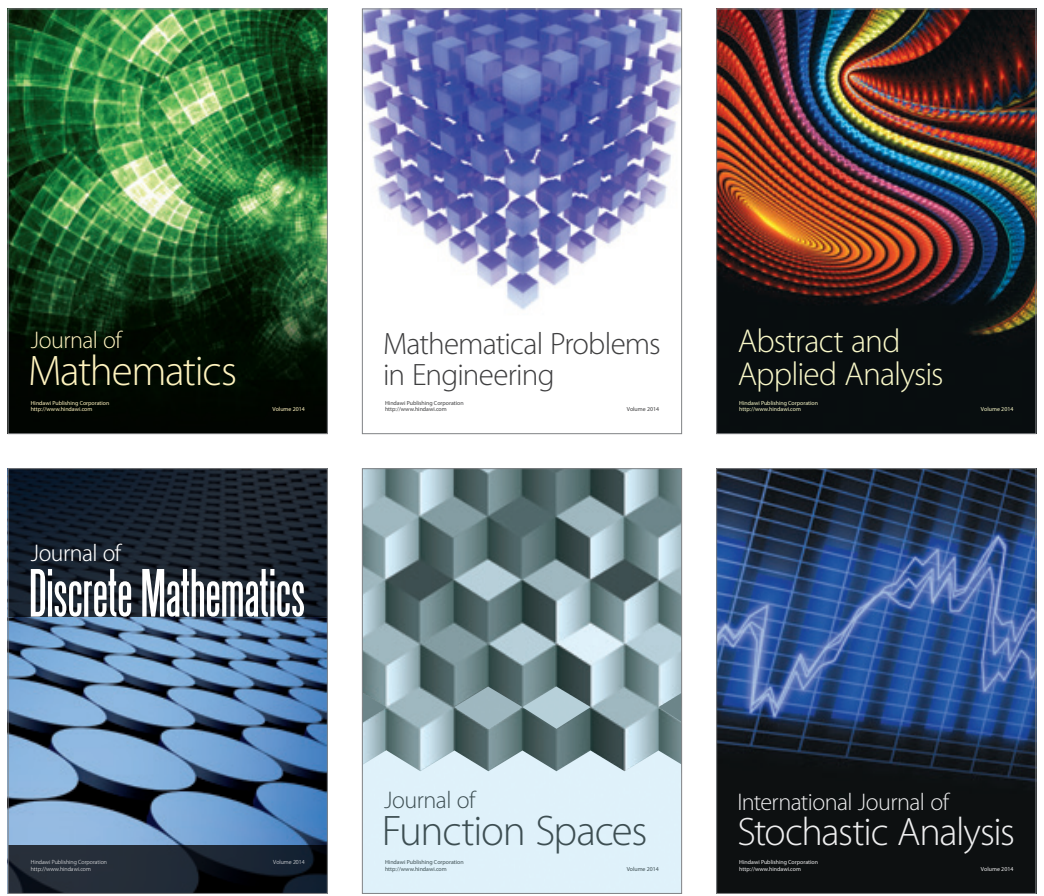

Journal of

Function Spaces

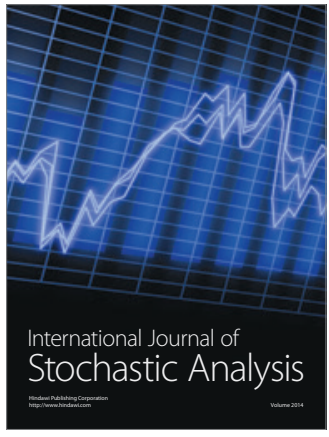

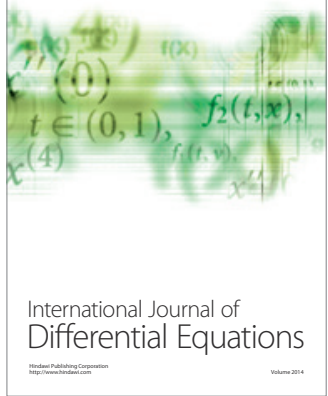
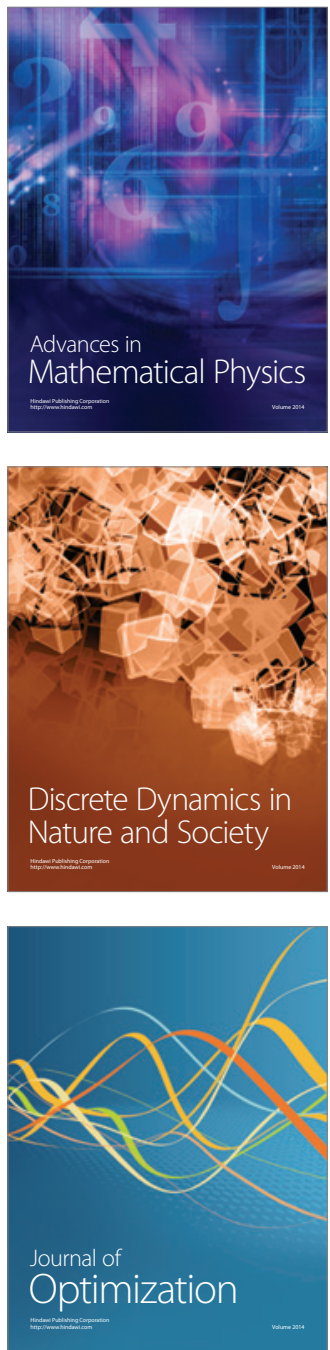